\title{
Medical Students and Interventional Radiology
}

\author{
Shyamkumar N. Keshava ${ }^{1}$ Alison Palumbo² \\ ${ }^{1}$ Department of Radiology, Christian Medical College, Vellore, Tamil \\ Nadu, India \\ ${ }^{2}$ Division of Interventional Radiology, Department of Radiology, \\ University of Texas Southwestern Medical Center, Dallas, Texas, \\ United States
}

J Clin Interv Radiol ISVIR 2018;2:1-1

A medical student is like a stem cell with potential to develop into any type of specialist in the medical field. Historically, medical students have had little exposure to the field of interventional radiology (IR) during their medical education and are often less familiar with the specialty as a career option.

The medical curriculum is rigorous, requiring medical students to master a wide breadth of basic medical science while learning the tenants of patient care. Medical imaging has been incorporated into the basic science curriculum over the last few years, and students are also exposed to the role radiology plays in the patient care during ward rounds. This underscores the role of imaging as an invaluable tool for guiding diagnosis and management. However, exposure to IR remains minimal.

Although the increased exposure to medical imaging is a move in the right direction, medical students need more and earlier exposure to IR. Regardless of whether or not they are interested in pursuing IR, learning about the role of an interventional radiologist and what procedures can be offered to their patients is integral to the medical curriculum. Exposure to IR can start on day 1 of medical school with IR faculty participating in specialty panel discussions for new medical students. Offering opportunities for medical students to shadow IR faculty, starting IR interest groups for medical students, and encouraging medical students to participate in IR procedures will also help increase awareness of this fascinating, complex specialty. Interactive, case-based lectures are not only an excellent learning tool but a great way for IR to showcase what IR has to offer to the patients. Showing emergent, lifesaving interventions for trauma, chemoembolization for hepatocellular carcinoma (HCC), as well as basic procedures such as central venous access will all help open their eyes to the exciting world of IR.

IR societies have generously supported medical students to attend IR conferences. As the resources to send students are limited, selection criteria to determine the level of interest in IR can be useful to determine which students will be selected. Students can not only participate in conferences via hands-on workshops and interactive sessions such as team quiz challenges but can also contribute academically with poster presentations. Some IR societies offer medical student sections and provide opportunities for students to participate on committees, further engaging them in the world of IR.

IR continues to develop into a more robust clinical subspecialty, with demand for our services growing in both procedure number and type. As a response to this change, IR has now become an independent residency pathway in the United States. Perhaps this change will eventually be adopted outside the United States.

It is important for IR as a specialty to make efforts to expose medical students early on to our exciting, dynamic subspecialty. This will both help raise awareness of what our specialty has to offer patients and get more medical students interested in pursuing IR as a career, all of which only help better our field and improve patient care.
Address for correspondence: Shyamkumar N. Keshava, MBBS, DMRD, DNB, FRCR, FRANZCR, Department of Radiology, Christian Medical College, Vellore 632004, Tamil Nadu, India (e-mail: aparna_shyam@cmcvellore.ac.in).
DOI https://doi.org/ 10.1055/s-0038-1649551. ISSN 2457-0214.
Copyright () 2018 Indian Society of Vascular and Interventional Radiology
License terms

() (1) $\ominus \circledast$ 